\title{
Recommendation System Based on Interaction with Multiple Agents for Users with Vague Intention
}

\author{
Itaru Kuramoto $^{1}$, Atsushi Yasuda ${ }^{1}$, Mitsuru Minakuchi $^{2}$, and Yoshihiro Tsujino ${ }^{1}$ \\ ${ }^{1}$ Kyoto Institute of Technology, Matsugasaki, Sakyo-ku, Kyoto 606-8585, Japan \\ ${ }^{2}$ Kyoto Sangyo University, Motoyama, Kamigamo, Kita-Ku, Kyoto 603-8555, Japan \\ entahit.is.kit.ac.jp
}

\begin{abstract}
We propose an agent-based recommendation system interface for users with vague intention based on interaction with multiple character agents, which are talking each other about their recommendations. This interface aims the user to make his/her intentions and/or potential opinions clear with hearing agents' conversation about recommendations. Whenever the user hits on any opinion, he/she can naturally join the conversation for getting more favorite recommendation. According to the result of experimental evaluation, the system with proposed interface can introduce more recommendations without any additional frustrations than the conventional recommendation systems with single agent.
\end{abstract}

Keywords: Vague intention, Character agent, Recommendation, Natural conversation.

\section{Introduction}

In the near future, there will be many recommendation systems, which recommend some items from a certain set of much information in order to support our decision making, and we will use such systems in common and frequently. In popular systems, we need to tell our intention and/or opinions to the system for getting recommendations. For example, we usually input some keywords to a bookrecommendation system for purchasing interesting books. Such a system forces us to tell explicit keywords related to our needs, but we sometimes have no clear intention or opinions about our needs. In such cases, we cannot make any keywords for telling to the system, so interaction with the system stops.

In case of casual communications among three or more people like chatting, such a problem rarely occurs. If some person has no clear words to speak about a current topic in chatting, he/she can be silent because the other people continue chatting. Moreover, the silent person may make his/her opinion clear during listening to the conversation of others, because there maybe some fruitful information about the current topic, which can stimulate for his/her imagination. When he/she hits on his/her opinion and wants to talk about it, he/she can naturally go back to the conversation kept by other chatting members.

In order to solve the problems of recommendation systems about users' unclear intention, we introduce the nature of the casual communication by three or more 
people. We propose an agent-based recommendation system interface which has two or more character agents who communicate with the user of the system for decision making. They talk each other if the user does not speak anything. The user can hear the conversation by the (two or more) agents, which includes some properties about some candidates of recommendation. The information can be some supports for his/her decision making. The user can rejoin to the conversation of them when he/she wants to say his/her opinion, so the user can take flexible communication to them.

\section{Related Work}

There are many recommendation systems with character agent interface. Moreover, there are some researches about the platform of conversational agent interface [1] so that agent-based recommendation systems will be appeared commonly in near future. However, most of such recommendation systems have only one agent [2].

Inhabited Market Place [3] is one of the agent-based presentation systems with multiple character agents collaborating with each other. In the system, the agents talk each other about an item (a car in [3]) for easy understanding about the item and for clarifying a user's unclear intention. Moreover, the user can interact to them with text input. The system is not a kind of recommendation systems, however, so it cannot change its presentation items for the user's preferences.

Sakamoto et al. proposed a competitive recommendation system with two character agents [4]. It is quite similar to our system. However, it focuses on the rationality of recommendation, so there are few discussions about interaction with agents and user. For example, it requires user's reactions ("Good" or "No good") at any ends of agents' speeches, so the user is forced to make a decision even if his/her opinion is unclear. Our study focuses on the aspect of natural interaction, so our system can avoid such situations.

Group recommender system is one of the instances of multiple character agent recommendation systems. Travel Decision Forum [5] is one of such systems. In the system, agents act a role of some members who is absent from a decision making meeting. The real members talk to the agents about the decision, and the agents tell opinions like as the absent members.

In addition, conversation between human and robot is one of the current topics [6]. It is known that the nature of human-robot interaction is different from that of humanscreen agent interaction [7]. It will be valuable to know the effect of our proposed recommendation method with robot as a representation of agent.

\section{Prototype}

We implement a prototype system with the proposed interface (shown in Figure 1). It recommends a restaurant for lunch near Kawaramachi station, which is one of the main stations in Kyoto, Japan. There are three character agents on the system, one is a moderator agent and two are competitor agents. A user interacts with them using three opinion buttons, that is, "I agree (your recommendation)", "I do not agree", and "Give more details" (see the bottom of Figure 1). 


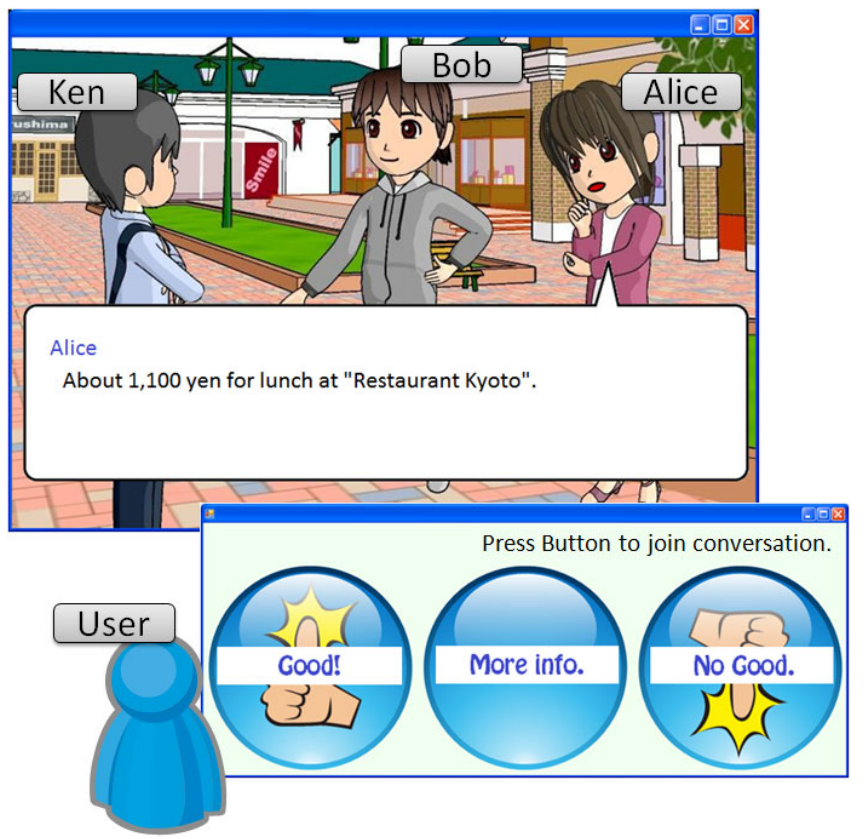

Fig. 1. Overview of restaurant recommendation system with multiple agents

\subsection{Algorithm}

At first, one of two competitor agents gives his/her recommendation to the user and the other agent. In case that the user does not push any buttons, which means he/she has no opinions about the recommendation, the other competitor agent gives his/her own recommendation. If the user does not push any buttons yet, the two agents continue to talk about their recommendations.

In the conversation, one competitor agent (named Alice below) tells about a certain property of Alice's recommendation, and then the other (named Bob below) replies Alice with saying the property of Bob's recommendation. For example, Alice says "My love restaurant is near, only 3 minutes far from here by walk", and Bob replies "Good. It takes 5 minutes from here to my recommendation", and so on. The moderator agent (named Ken below) prompts them in order to keep natural and smooth conversation, and does not tell any opinions eventually.

If the user pushes "I agree" button during Alice is saying something, which means he/she decides to follow Alice's recommendation, Ken asks him/her to confirm, and then the recommendation process is finished. On the other hand, if the user pushes "I do not agree" button during Bob is speaking, Bob withdraws his opinion and recommend another new restaurant. If the user pushes "Give more details" button, the speaker (Alice or Bob) talks about another property of his/her recommendation.

In case of no user's decision and no more detail information for chat, Ken selects one of their recommendations (randomly in the prototype system) and asks the user to confirm it. If the user disagrees it, the agent who recommends it changes his/her recommendation and then return chatting. If the user does not reply again, Ken recommend the selected one and finish the recommendation process. 


\subsection{Implementation}

The prototype system has the information of 156 restaurants which are less than $1.0 \mathrm{~km}$ from Kawaramachi station. The information includes the name, map, photo and properties of the restaurants such as genre, average cost, distance (by minutes' walk), and chef's recommendation menu. In this prototype system, the first recommendations by Alice and Bob are randomly selected.

The conversation is shown by text. Only one speech of a certain agent is shown at one time, so there is a 3.0-second space between the end of a certain agent's speech and the start of the next agent's speech in order that the user can catch up the conversation and tell his/her opinion by the opinion buttons.

Alice and Bob have sub-windows one for each (shown in Figure 2). It displays the name, map and photo of currently recommended restaurant by Alice or Bob. A matrix barcode on the upper-right of the window is the URL of the restaurant's website (if it has). A user can easily get the information of the recommended restaurant with his/her feature phone.

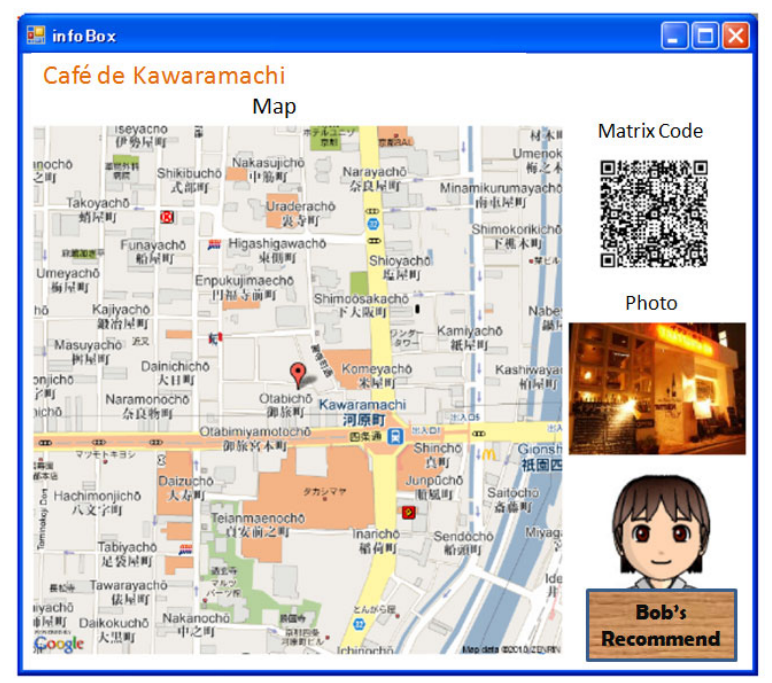

Fig. 2. Sub-window for current recommendation

\section{Evaluation}

\subsection{Method}

We asked 12 participants to evaluate the proposed system by comparing it to the conventional one shown in Figure 3. The conventional system has only one agent (named Charlie below). The system has the same interaction style (three buttons) as the proposed one.

In the conventional system, Charlie says some properties of his recommendation, and then the user decides whether he/she agrees Charlie's recommendation or not, or requests some more details for decision with three buttons. 
In the evaluation, we ask the participants to use either conventional or proposed system to decide the restaurant for the day's lunch, and at the next day they use another one. We measured the number of recommended restaurants and the time of usage. After the two usages of recommendation systems, we asked participants to answer the questions below:

- Q1: Which system do you feel easy to make decision?

- Q2: Which system's recommendation do you think better?

- Q3: Which system do you feel boring?

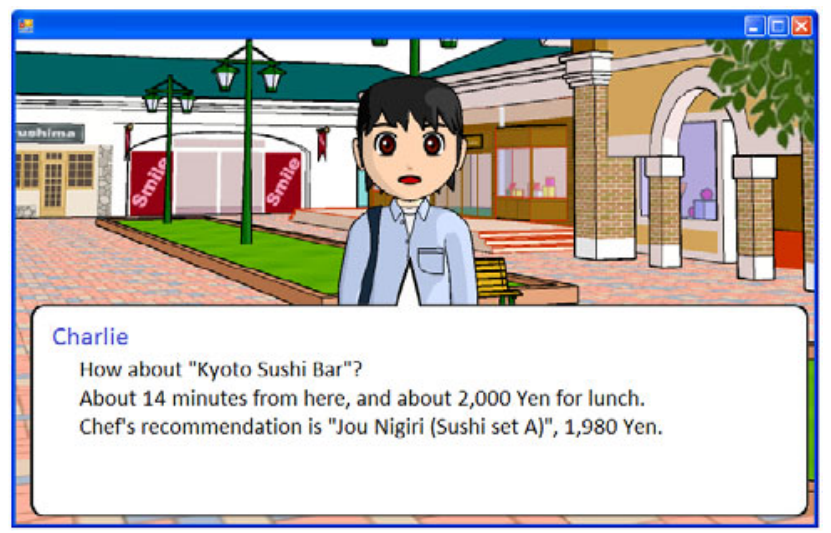

Fig. 3. Conventional system (with single agent)

\subsection{Result}

Figure 4 shows the result of the average time of recommendation (a) and the average number of restaurants recommended (b). As a result, the time for interaction with the proposed system is longer than with the conventional one. However, the proposed system could introduce more recommendations to the participants than the conventional one.

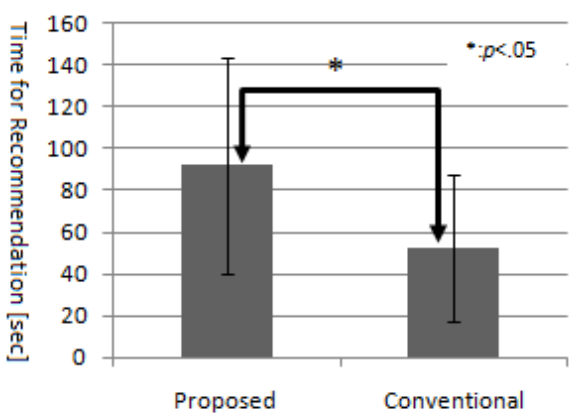

(a)

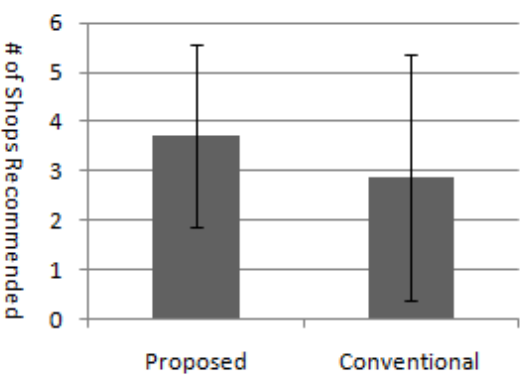

(b)

Fig. 4. The result of evaluation: (a) the average time for recommendation, (b) the average number of restaurants recommended. 
Table 1. The result of questions after using recommendation system

\begin{tabular}{|l|r|r|r|}
\hline & Proposed & Conventional & \multicolumn{1}{|c|}{ Same } \\
\hline Q1 & 3 & 5 & 4 \\
\hline Q2 & 2 & 5 & 5 \\
\hline Q3 $^{*}$ & 1 & 9 & 2 \\
\hline
\end{tabular}

Table 1 shows the result of questions after the usages of recommendation systems. The result of Q3 indicates that recommendations with the proposed method give no additional frustrations even though the time for recommendation is significantly longer. It means that the system has a potential for supporting preferable decision making for users.

By contrast, the results of Q1 and Q2 indicate that the participants satisfy slightly more with the conventional system than the proposed one. In interviews after the evaluation, some participants pointed out that they preferred the conventional system because they could think deeply about the restaurant recommended. The proposed system cannot stop the conversation because of giving more information for users without explicit intention or opinions, so there was not enough time for them to make decision with deep thought. The function of reviewing conversations is needed for solving such a problem.

\section{Conclusion and Future Work}

We proposed an agent-based recommendation system interface which has two or more character agents who communicate with the user of the system for decision making. They talk each other about their recommendations, and the user can join and tell his/her opinions whenever he/she like, such as when his/her intention becomes clear by their conversation.

As a result of experimental evaluation, the time for recommendation with the proposed system is longer than with the traditional one. However, the proposed system could introduce more recommendations to the participants than the traditional one with no additional frustrations. It indicates that the participants can make more precise decision.

In future, we are planning to introduce characteristics into the agents. Individuality of character agents is one of the important aspects for smooth and natural interaction with human [8]. We believe it leads that conversations with the agents is more smooth and interesting.

\section{References}

1. Rist, T., Andre, E., Baldes, S.: A flexible Platform for Building Applications with Life-Like Characters. In: the 8th International Conference on Intelligent User Interfaces (IUI 2003), pp. 158-165. ACM, New York (2003)

2. Shimazu, H.: ExpertClerk: Navigating Shoppers' Buying Process with the Combination of Asking and Proposing. In: 7th International Joint Conference on Artificial Intelligence, pp. 1443-1448 (2001) 
3. André, E., Rist, T.: Adding Life-Like Synthetic Characters to the Web. In: Klusch, M., Kerschberg, L. (eds.) CIA 2000. LNCS (LNAI), vol. 1860, pp. 1-13. Springer, Heidelberg (2000)

4. Sakamoto, T., Kitamura, Y., Tatsumi, S.: A Competitive Information Recommendation System and Its Rational Recommendation Method. Systems and Computers in Japan 38(9), 74-84 (2007)

5. Jameson, A.: More than the Sum of Its Members: Challenges for Group Recommendation Systems. In: The Working Conference on Advanced Visual Interfaces (AVI 2004), pp. 4854. ACM, New York (2004)

6. Mutlu, B., Shiwa, T., Kanda, T., Ishiguro, H., Hagira, N.: Footing in Human-Robot Conversations: How Robots might Shape Participant Roles Using Gaze Cues. In: the Fourth ACM/IEEE International Conference on Human-Robot Interaction (HRI 2009), pp. 61-68. ACM, New York (2009)

7. Shinozawa, K., Naya, F., Yamato, J., Kogure, K.: Differences in effect of robot and screen agent recommendations on human decision-making. International Jounal of HumanComputer Studies 62, 267-279 (2005)

8. Isard, A., Brockmann, C., Oberlander, J.: Individuality and Alignment in Generated Dialogues. In: The Fourth International Natural Language Generation Conference, pp. 2532. Association for Computational Linguistic, Sydney (2006) 